EPJ Web of Conferences 38, 10003 (2012)

DOI: 10.1051 epjconf/20123810003

(C) Owned by the authors, published by EDP Sciences, 2012

\title{
Level lifetimes and quadrupole moments from Coulomb excitation in the Ba chain and the $\mathbf{N}=80$ isotones
}

\author{
C. Bauer ${ }^{1, a}$, G. Guastalla ${ }^{1}$, J. Leske ${ }^{1}$, O. Möller ${ }^{1}$, T. Möller ${ }^{1}$, J. Pakarinen ${ }^{2}$, N. Pietralla ${ }^{1,3}$, G. Rainovski ${ }^{4}$, E. Rapisarda $^{2}$, \\ D. Seweryniak ${ }^{5}$, C. Stahl ${ }^{1}$, R. Stegmann ${ }^{1}$, J. Wiederhold ${ }^{1}$, and S. Zhu ${ }^{5}$ \\ 1 Institut für Kernphysik, Technische Universität Darmstadt, D-64289 Darmstadt, Germany \\ 2 CERN, Genève, Switzerland \\ 3 GSI Helmholtzzentrum für Schwerionenforschung mbH, D-64291 Darmstadt, Germany \\ 4 Faculty of Physics, St. Kliment Ohridski University of Sofia, BG-1164 Sofia, Bulgaria \\ 5 Argonne National Lab, Chicago, Illinois, U.S.A.
}

\begin{abstract}
The chain of Barium isotopes enables us to study experimentally the evolution of nuclear quadrupole collectivity from the shell closure at $N=82$ towards neutron-deficient or neutron-rich deformed nuclei. The TU Darmstadt group has investigated several nuclei from stable ${ }^{130,132} \mathrm{Ba}$ up to radioactive ${ }^{140,142} \mathrm{Ba}$ with the projectile-Coulomb excitation technique including the use of the Doppler-shift attenuation method (DSAM). Lifetimes of quadrupole-collective states of ${ }^{132} \mathrm{Ba}$ and ${ }^{140} \mathrm{Ba}$ were obtained for the first time as well as the static electric quadrupole moments $Q\left(2_{1}^{+}\right)$for ${ }^{130,132} \mathrm{Ba}$ and ${ }^{140,142} \mathrm{Ba}$. The results are compared to Monte Carlo shell model and Beyond-Mean-Field calculations. The phenomenon of shell stabilization in the $N=80$ isotones is further investigated by measurements of the $\mathrm{B}\left(\mathrm{E} 2 ; 2_{1}^{+} \rightarrow 0_{1}^{+}\right)$values of ${ }^{140} \mathrm{Nd}$ and ${ }^{142} \mathrm{Sm}$ and comparison to the quasi-particle phonon model and shell-model calculations.
\end{abstract}

\section{Introduction}

The properties of the ground states and low-lying excited states of many atomic nuclei can be attributed to the correlated or collective motion of their nucleons in terms of surface oscillations or the rotation of a permanently deformed spatial distribution. They reflect the structural changes when progressively more particles or holes are added to closed-shell nuclei, at first softening the (spherical) nuclear potential and then resulting in a deformed equilibrium shape.

The isotopic chain of the even-N Ba isotopes exhibits rather peculiar patterns of structural evolution. When neutrons are successively removed from the $N=82$ neutronshell closure, the collectivity of $\mathrm{Ba}$ isotopes increases and drives their shape from spherical towards considerable deformation passing a transitional region with soft triaxiality [1]. In the neutron-rich $\mathrm{Ba}$ isotopes with $\mathrm{N} \geq 82$ the semi-magic ${ }^{138} \mathrm{Ba}$ is spherical, whereas ${ }^{148} \mathrm{Ba}$ has a $R_{4 / 2}=$ $E\left(4_{1}^{+}\right) / E\left(2_{1}^{+}\right)$ratio of $\sim 2.98$, closer to a deformed axiallysymmetric rotor with an $R_{4 / 2}$ value exceeding the one for the $\mathrm{X}(5)$ scheme [2].

There is evidence for octupole correlations in the mass region around $A \sim 146(Z=56, N \sim 90)$ that may influence the properties of nuclei already at low excitation energies since the resulting gain in binding energy is sufficient to stabilize permanent octupole deformations [3, 4]. Still, low-lying energy levels with positive parity and

\footnotetext{
a e-mail: bauer@ikp.tu-darmstadt.de
}

electrical quadrupole transition probabilities of neutronrich $\mathrm{Ba}$ isotopes have been successfully described throughout the transitional region in the framework of the Monte Carlo shell model based on a quadrupole-plus-pairing approach $[5,6]$.

Since in many nuclei quadrupole deformation predominates [7], a key observable to characterize the intrinsic nuclear shape in an even-even nucleus is the static quadrupole moment of the $2_{1}^{+}$state used in conjunction with a collective model [8]. Together with the E2 transition rates it provides a sensitive tool for characterizing spherical-todeformed shape transitions. Because for lifetimes below $\sim 10 \mathrm{~ns}$ no direct method is available to determine the static quadrupole moment of excited nuclear states, Coulomb excitation cross-sections, reduced electrical transition probabilities and lifetimes have to be used in order to deduce the spectroscopic quadrupole moment and nuclear deformation of these states [9].

\section{Experimental methods}

\subsection{Doppler-shift attenuation method (DSAM)}

For lifetimes of nuclear states of a few picoseconds (ps) down to some 10 femtoseconds (fs), the Doppler-Shift Attenuation Method (DSAM) can be applied [10]. In general the investigated state of a nucleus moving at a certain velocity (e.g. of the projectile in a Coulomb excitation experiment) is populated and the deexcitation occurs during the 


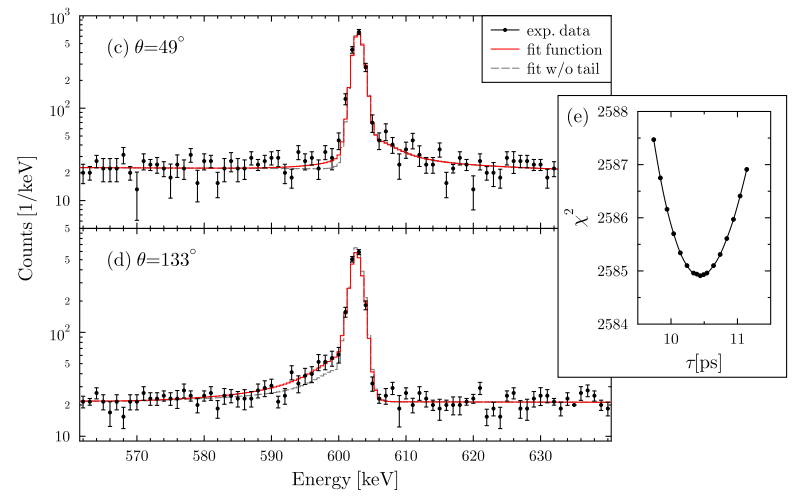

Fig. 1. The Doppler-broadened lineshapes of the $2_{1}^{+} \rightarrow 0_{1}^{+}$transition in ${ }^{140} \mathrm{Ba}$ for (c) $49^{\circ}$ and (d) $133^{\circ}$ are shown after backgroundsubtraction. The spectra are shifted by +20 counts per channel for better visibility on the logarithmic scale. The full fits are represented by solid (red) lines, the corresponding peak shapes not corrected for the effect of neutron damage as obtained from longlived transitions in the same spectra are dashed (grey). At forward angles the Doppler-lineshapes are not significantly disturbed by any low-energy tailing due to partial neutron damage of the $\mathrm{Ge}$ detectors. Panel (e) shows the $\chi^{2}$ values for the combined fit to the lineshapes detected in 18 crystals as a function of the assumed lifetime of the $2_{1}^{+}$state $\left(\chi_{\min }^{2} / N O F=0.92\right)$. This figure is an updated version of figure 2 in Ref. [6].

deceleration of the ion in a thick target. A characteristic, Doppler-broadened photopeak from the $\gamma$ rays depopulating the short-lived nuclear state can be detected under forward or backward direction. The distinct lineshape of this peak is determined by the lifetime of the state.

We have developed a new approach for analyzing DSA data taken with the new generation of position-sensitive $\gamma$-ray detector arrays coming, such as AGATA [11] or GRETA [12]. It takes advantage of the feature that the spectra of the $\gamma$-ray transitions are almost continuous in energy and observation angle. The analysis of these twodimensional spectra in energy and polar angle of the observation point represents an advancement of the conventional DSA method which is based on the analysis of onedimensional spectra recorded under discrete detector positions and averaged over the solid angle covered by the detectors. The new approach enhances the sensitivity, efficiency, and applicability of the DSA method [13]. Fits of the lineshapes in the energy-angle space, that simultaneously contain information collected under all polar angles covered by the detector array, promise a significant improvement of the accuracy of the lifetimes obtained as well as extending the applicability to cases that were not accessible by conventional analysis due to non-trivial contaminant lineshapes or low statistics. The capability to determine lifetimes also from spectra with low statistics is of major interest especially for experiments with radioactive ion beams and/or reactions with low excitations probabilities for the desired states.

As a user-friendly tool for this kind of analysis the software APCAD [14] was developed. It uses stopping matrices describing the Coulomb excitation and slowing-down process of the heavy ions in the target calculated from a Monte-Carlo simulation based on the GEANT4 framework [15] using the stopping powers from [16] and [17, 18]. In order to estimate the consequence of uncertainties in the used stopping power method, we perform fits with stopping-matrices obtained with scaled stopping powers. For the presented result of ${ }^{140} \mathrm{Ba}$ the stopping powers have been varied by $\pm 5 \%$ for the electronic stopping and $\pm 20 \%$ for the nuclear stopping, thus reflecting their increasing uncertainty at low ion velocities.

Although current quasi- $4 \pi-\gamma$-ray detector arrays do not yet provide continuous-angle $\gamma$-spectra they cover a wide range of discrete angles either ordered in rings of polar angles (Gammasphere [19]) or by specific positions of the detector cores (MINIBALL [20]). This makes it still possible to use the the angular information in the APCAD software by not fitting individual spectra separately but connecting them in a simultaneous analysis via the angular distribution of the investigated state. This was done in the analysis of the lifetime $\tau\left(2_{1}^{+}\right)$of ${ }^{140} \mathrm{Ba}$ [6], e.g., where the radioactive ion beam was stopped in a thick ${ }^{n a t} \mathrm{Cu}$ target and the $\gamma$-rays were detected by MINIBALL (cf. figure 1).

\subsection{Reorientation technique}

In particular, the reorientation technique [21] can be used for the measurement of the spectroscopic quadrupole moment. It makes use of the fact that in Coulomb excitation experiments apart from the transitional matrix element $M_{20}=\left\langle 0_{1}^{+}\|M(E 2)\| 2_{1}^{+}\right\rangle$the cross section $\sigma\left(2_{1}^{+}\right)$depends, to a smaller extent, also on the diagonal matrix element $M_{22}=\left\langle 2_{1}^{+}\|M(E 2)\| 2_{1}^{+}\right\rangle$. The latter is related to the spectroscopic quadrupole moment: $Q\left(2_{1}^{+}\right)=0.758 M_{22}$. Therefore, the Coulomb excitation yield can be used to extract information on the quadrupole moment $Q\left(2_{1}^{+}\right)$. The relation between the $\mathrm{B}(\mathrm{E} 2)$ value and the lifetime $\tau$ obtained from an independent measurement has then to be used to constrain the value of the $M_{20}$ matrix element sufficiently precisely for such a Coulomb yield analysis. The importance of precise lifetime information as a sine qua non for the interpretation of data from reorientation experiments has been demonstrated recently when determining the sign of the spectroscopic quadrupole moment of the $2_{1}^{+}$state of ${ }^{70}$ Se $[22,23]$.

The experimental data of projectile Coulomb excitation was taken at two facilities, namely at the Argonne National Lab and at CERN. Ion beams of the rare stable isotopes ${ }^{130,132} \mathrm{Ba}$ with a natural abundance of about $0.1 \%$ were produced by the ATLAS facility at ANL. The $\gamma$-rays from Coulomb excitation reactions were detected by the Gammasphere spectrometer. The measurements with the radioactive isotopes ${ }^{140} \mathrm{Ba}\left(T_{1 / 2}=12.75 \mathrm{~d}\right),{ }^{142} \mathrm{Ba}$ $\left(T_{1 / 2}=10.7 \mathrm{~m}\right)$ and ${ }^{140} \mathrm{Nd}\left(T_{1 / 2}=3.37 \mathrm{~d}\right)$ as well as ${ }^{142} \mathrm{Sm}$ $\left(T_{1 / 2}=72.4 \mathrm{~m}\right)$ took place at the REX-ISOLDE facility at CERN using the MINIBALL array. For ${ }^{130,132} \mathrm{Ba}$ data sets with a $0.462 \mathrm{mg} / \mathrm{cm}^{2}$ thick ${ }^{94}$ Mo target were taken at two different energies for each of the isotopes to disentangle the interference effect on the quadrupole moment (see 
Ref. [24] for ambiguous data of $Q$ moments in the Ba isotopes). The radioactive nuclei ${ }^{140,142} \mathrm{Ba}$ were Coulomb excited on a $0.9 \mathrm{mg} / \mathrm{cm}^{2}$ thick target of ${ }^{96} \mathrm{Mo}$ [6]. The ${ }^{140} \mathrm{Nd}$ beam was impinging on 2 different targets for Coulomb excitation $\left({ }^{48}\right.$ Ti with $1.4 \mathrm{mg} / \mathrm{cm}^{2}$ and ${ }^{64} \mathrm{Zn}$ with $\left.1.55 \mathrm{mg} / \mathrm{cm}^{2}\right)$ to determine the transitions strength $\mathrm{B}\left(\mathrm{E} 2 ; 2_{1}^{+} \rightarrow 0_{1}^{+}\right)$. For the same reason ${ }^{142} \mathrm{Sm}$ was investigated via scattering off ${ }^{48} \mathrm{Ti}$ with $1.4 \mathrm{mg} / \mathrm{cm}^{2}$ and ${ }^{94} \mathrm{Mo}$ with $2.0 \mathrm{mg} / \mathrm{cm}^{2}$.

\section{Evolution of quadrupole moments $Q\left(2_{1}^{+}\right)$ in the Barium chain}

The uncertainty of the previous literature value of $\tau=$ $14.0 \pm 5.9 \mathrm{ps}$ for the lifetime of the $2_{1}^{+}$state of ${ }^{140} \mathrm{Ba}$ [25] did not permit the use of the reorientation analysis. Therefore a complementary lifetime measurement needed to be performed using DSAM. In order to efficiently use the precious beam time for intense radioactive beams and for minimizing systematical errors, it is desirable to do both measurements using one and the same setting of the radioactive beam. This approach differs from preceding experiments at MINIBALL applying the reorientation method with Coulomb excitation reactions [22,26]. Ref. [6] reports on this measurement of the sign and magnitude of the spectroscopic quadrupole moment of the $2_{1}^{+}$state of neutron-rich unstable ${ }^{140} \mathrm{Ba}$ using a unique combination of the reorientation technique and the DSA method for the same setting of the radioactive beam for the first time.

Beside ${ }^{140} \mathrm{Ba}$, the neighboring even-even nucleus ${ }^{142} \mathrm{Ba}$ [27] was measured in the same campaign. Here a previously measured lifetime of good accuracy [25] allowed for a straightforward reorientation analysis. The stable isotopes ${ }^{130,132} \mathrm{Ba}$ have been measured at the Gammasphere setup as explained in Subsec. 2.2. Preliminary results

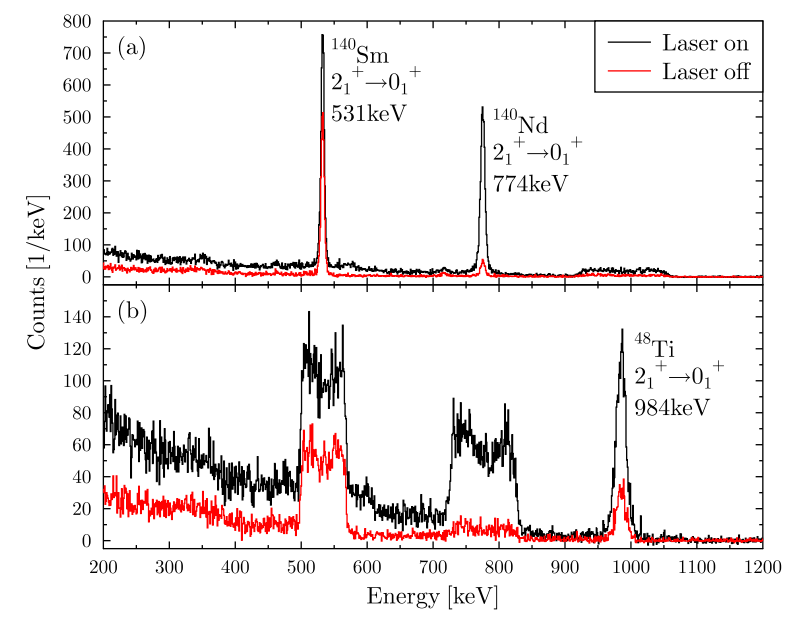

Fig. 2. Background-subtracted particle- $\gamma$ coincidence spectra applying Doppler-correction with respect to the (a) projectile or (b) recoiling target nuclei showing the only observed transitions, namely the $2_{1}^{+} \rightarrow 0_{1}^{+}$transitions in ${ }^{140} \mathrm{Sm}$ at $531 \mathrm{keV}$, in ${ }^{140} \mathrm{Nd}$ at $774 \mathrm{keV}$ and in ${ }^{48} \mathrm{Ti}$ at $984 \mathrm{keV}$. The data with laser ionization switched on (black) and off (red) is shown in the same spectrum.

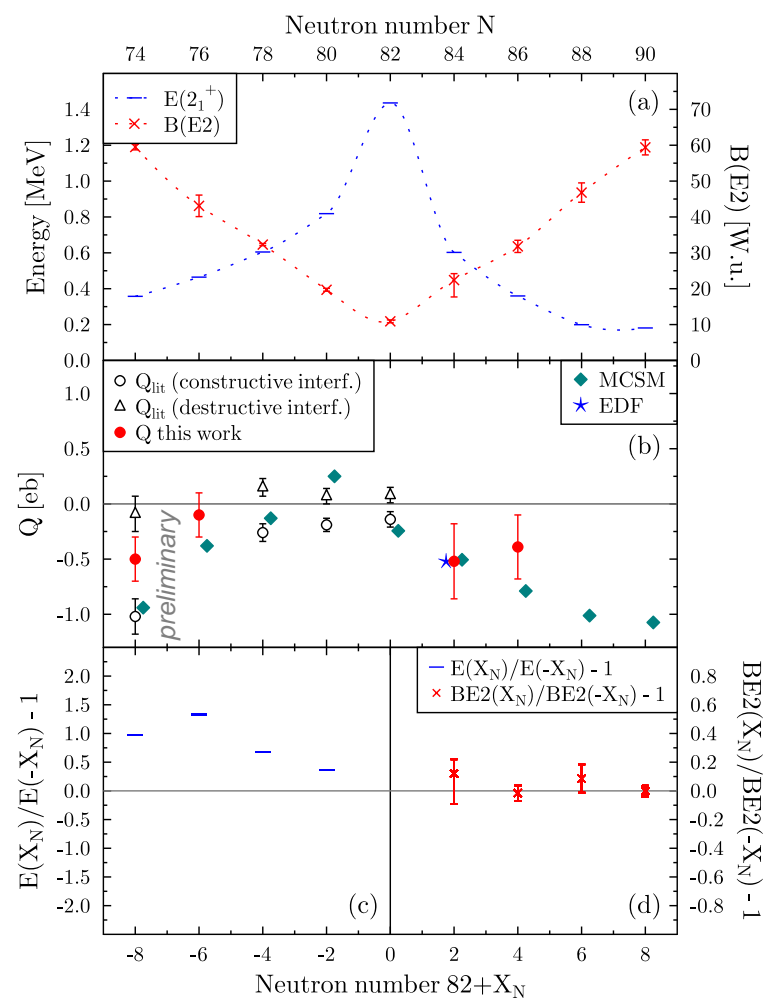

Fig. 3. Systematics of excitation energies and $B(E 2)$ values in W.u. (a) and $Q$ moments (b) for the first excited $2_{1}^{+}$states in the even $\mathrm{Ba}$ isotopes with $130 \leq \mathrm{A} \leq 146$. Panel (b) is updated from figure 5 of Ref. [6]. The ratio $E\left(2_{1}^{+} ; 82-X_{N}\right) / E\left(2_{1}^{+} ; 82+X_{N}\right)-1$ is shown (c) and analogously $B\left(E 2 ; 82+X_{N}\right) / B\left(E 2 ; 82-X_{N}\right)-$ 1 (d) to highlight the specific mirror symmetry of the transition strengths around $N=82$. All B(E2) values are given in W.u. in order to account for a simple size-effect.

are summarized in figure 3 showing also the distinct symmetry of transition strength in the Ba isotopes (cf. figure 3 (a) and (c)). The preliminary quadrupole moments (middle panel (b) of figure 3) agree with recent Monte-Carlo shell-model calculations [28,29]. Different model spaces are used for this calculations when the neutron number $N$ is above or below the shell closure of $N=82$. For $Z=50 \sim 82$ and $N=82 \sim 126$ the effective charges are $\left(e_{p}, e_{n}\right)=(1.6,0.6) e$ while for $Z=50 \sim 82, N=82 \sim 50$ they are $\left(e_{p}, e_{n}\right)=(1.5,1.0) e$. In the case of ${ }^{140} \mathrm{Ba}$ energy density functional calculations using the Gogny D1S force (cf. figure 3 and Ref. [6]) are confirmed by the measurement of the quadrupole moment.

\section{Sudden jump of $B\left(E 2 ; 2_{1}^{+} \rightarrow 0_{1}^{+}\right)$strength in the $\mathbf{N}=\mathbf{8 0}$ isotones}

Recent measurements at the REX-ISOLDE facility allowed to extend the systematics of $\mathrm{B}\left(\mathrm{E} 2 ; 2_{1}^{+} \rightarrow 0_{1}^{+}\right)$strength in the $N=80$ isotones (see also Refs. $[30,31]$ ) above the $Z=58$ subshell closure for neutrons and going to radioactive nuclei. The preliminary results of $\geq 30$ W.u. for ${ }^{140} \mathrm{Nd}$ 
and for ${ }^{142} \mathrm{Sm}$ are shown in comparison with the available data in figure 4 . The trend of growing $\mathrm{B}\left(\mathrm{E} 2 ; 2_{1}^{+} \rightarrow 0_{1}^{+}\right)$ strength in the $N=80$ isotones while moving away from the $Z=50$ shell closure is expected due to increasing collectivity and also described by the empirical Grodzins relation (cf. figure 4). Recent calculations within the quasiparticle phonon model (QPM) for the $N=80$ isotones [38] predict a smaller $\mathrm{B}\left(\mathrm{E} 2 ; 2_{1}^{+} \rightarrow 0_{1}^{+}\right)$value compared to the preliminary data from experiments. Recent large-scale shell-model calculations [37] predict higher strengths. In the shell model calculations a realistic Hamiltonian $(\mathrm{G}-$ matrix derived from the CD-Bonn potential) is used with the same single particle energies and the same effective charges for all of the isotones as well as for the Xenon isotopes [40].

The QPM as well as the shell-model calculations provide a good agreement of the excitation energies for the $2_{x}^{+}$states (cf. figure 5). Nevertheless, the strong increase in transition strength for ${ }^{140} \mathrm{Nd}$ cannot be explained by any of the two models. On the other hand the energies of the $2_{1}^{+}$states and the suspected $2_{m s}^{+}$states in ${ }^{140} \mathrm{Nd} \mathrm{[41]} \mathrm{and}$ ${ }^{142} \mathrm{Sm}$ agree well with the presented fit of a 2-state mixing in Ref. [31].

\section{Summary}

Quadrupole moments of excited $2_{1}^{+}$states have been measured in ${ }^{130,132,140,142} \mathrm{Ba}$. Beside the classical reorientation technique we have utilized a new combined technique of lifetime measurement at REX-ISOLDE and MINIBALL using the Doppler-shift attenuation method (DSAM) and a reorientation analysis of Coulomb excitation yields. The findings for the quadrupole moments in the Ba iso-

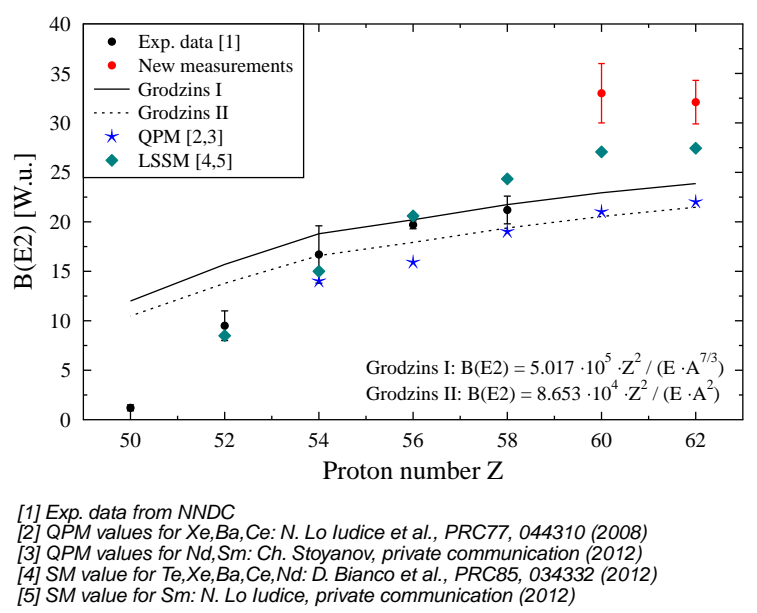

Fig. 4. Systematics of $B(E 2)$ values in W.u. for the first excited $2_{1}^{+}$states in the even $N=80$ isotones from $Z=50$ (Sn) to $Z=62$ $(\mathrm{Sm})$. The existing experimental data [32-36] is presented including our new values for ${ }^{140} \mathrm{Nd}$ and ${ }^{142} \mathrm{Sm}$ in comparison with large-scale shell-model calculations [37] as well as the quasiparticle phonon model $[38,39]$. Two representations of the empirical Grodzins relation are shown as well.

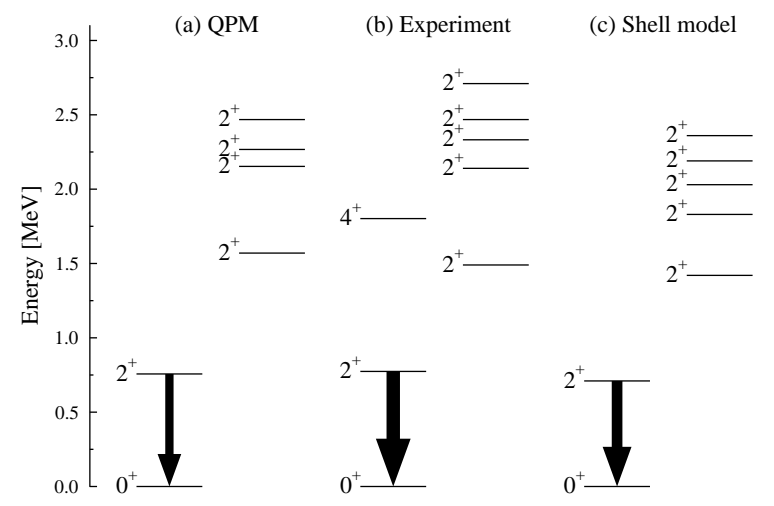

Fig. 5. The experimental level scheme for ${ }^{140} \mathrm{Nd}(\mathrm{b})$ is shown together with the calculated ones from the (a) QPM [39] and (c) shell model [37]. The illustration concentrates on the $2_{x}^{+}$states with the transition strength of the $2_{1}^{+}$state to the g.s. represented by the thickness of the arrows.

topic chain are in agreement with results from the Monte Carlo shell-model approach and were in the case of ${ }^{140} \mathrm{Ba}$ tested explicitly with beyond-mean-field calculations (energy density functionals) using the Gogny D1S force.

The trend of growing $\mathrm{B}\left(\mathrm{E} 2 ; 2_{1}^{+} \rightarrow 0_{1}^{+}\right)$strength in the $N=80$ isotones while moving away from the $Z=50$ shell closure is expected due to higher collectivity but the surprising increase beyond the $Z=58$ subshell $\left({ }^{140} \mathrm{Nd}\right.$ and ${ }^{142} \mathrm{Sm}$ ) is not entirely understood.

\section{Acknowledgements}

We thank all those who contributed to our experiments at ANL and REX-ISOLDE. For the interpretation of the data, we thank N. Shimizu, T. Otsuka, T.R. Rodríguez, Ch. Stoyanov, D. Tarpanov, N. Lo Iudice, and D. Bianco. We gratefully acknowledge the support of the scientists and staff members at the mentioned labs during the experimental runs. In particular, we thank the IS411 collaboration (see Ref. [6]) for providing us with the data on ${ }^{140,142} \mathrm{Ba}$ and T. Stora, D. Voulot, and F. Wenander for their continuous support during all experiments at MINIBALL. The data analysis of the experiments on ${ }^{140,142} \mathrm{Ba}$ and the experiments on ${ }^{140} \mathrm{Nd},{ }^{142} \mathrm{Sm}$ have been supported by the BMBF under grant Nos. 06DA9036I, 06DA9041I, 06DA7046, 06MT238, 06MT9156, RII3-EURONS (Contract No. 506065) and by HIC for FAIR funded by the State of Hesse in the framework of the LOEWE program. The experiments on ${ }^{130,132} \mathrm{Ba}$ were supported by the DFG under grant No. Pi 393/2-2 and the German-Bulgarian exchange program under grant Nos. PPP 50751591 and DNTS/01/2/2011.

\section{References}

1. R.F. Casten, Nuclear Structure from a Simple Perspective (Oxford University Press, Oxford 2000) 
2. F. Iachello, Phys. Rev. Lett. 87, 052502 (2001)

3. W.R. Phillips, et al., Phys. Rev. Lett. 57, 3257 (1986)

4. P.A. Butler and W. Nazarewicz, Rev. Mod. Phys. 68, No. 2 (1996)

5. N. Shimizu, T. Otsuka, T. Mizusaki, M. Honma, Phys. Rev. Lett. 86, 1171 (2001)

6. C. Bauer, et al., Phys. Rev. C 86, 034310 (2012)

7. P. Ring and P. Schuck, The Nuclear Many-Body Problem (Springer Verlag, Berlin 1980)

8. B. Castel and I.S. Towner, Modern Theories of Nuclear Moments (Clarendon Press, Oxford 1990)

9. G. Neyens, Rep. Prog. Phys. 66, 633 (2003)

10. T. K. Alexander and J. S. Foster, Nucl. Phys. A 10, 197 (1979)

11. S. Akkoyun, et al., Nucl. Instr. Meth. A 668, 26 (2012)

12. M. Deleplanque, I. Lee, K. Vetter, G. Schmid, F. Stephens, R. Clark, R. Diamond, P. Fallon, and A. Macchiavelli, Nucl. Instr. Meth. A 430, 292 (1999)

13. C. Stahl, Continuous-Angle DSAM: A new quality of lifetime measurements, Master Thesis (TU Darmstadt 2011)

14. C. Stahl, Software package APCAD, to be published (2012)

15. S. Agostinelli, et al., Nucl. Instr. and Meth. A 506, 250 (2003)

16. Physics reference manual, version: geant4.9.4 (http://geant4.web.cern.ch/geant4/support/)

17. ICRU report 49, Journal of the ICRU (1993)

18. ICRU report 73: Stopping of ions heavier than helium, Journal of the ICRU (2005)

19. I.-Y. Lee, Nucl. Phys. A 520, 641 (1990)

20. P. Reiter, et al., Nucl. Phys. A 701, 209 (2002)

21. J. de Boer, et al., Phys. Rev. Lett. 14, 564 (1965)

22. A.M. Hurst, et al., Phys. Rev. Lett. 98, 072501 (2007)

23. J. Ljungvall, et al., Phys. Rev. Lett. 100, 102502 (2008)

24. S. Burnett, A. Baxter, G. Gyapong, M. Fewell, and R. Spear, Nucl. Phys. A 494, 102 (1989)

25. H. Mach, R.L. Gill, M. Moszynski, Nucl. Instr. Meth. Phys. Res. A 280, 49 (1989)

26. A. Ekstrom, et al., Phys. Rev. C 80, 054302 (2009)

27. R. Stegmann, Investigation of the first $2^{+}$state and its deformation in radioactive ${ }^{142} \mathrm{Ba}$ by Coulomb excitation, Bachelor Thesis (TU Darmstadt 2011)

28. N. Shimizu, T. Otsuka et al., Phys. Rev. Lett. 86, 1171 (2001)

29. N. Shimizu, private communication (2012)

30. G. Rainovski, N. Pietralla, T. Ahn, C. J. Lister, R. V. F. Janssens, M. P. Carpenter, S. Zhu, and C. J. Barton, Phys. Rev. Lett. 96, 122501 (2006)

31. T. Ahn, L. Coquard, N. Pietralla, G. Rainovski, A. Costin, R.V.F. Janssens, C.J. Lister, M. Carpenter, S. Zhu, and K. Heyde, Phys. Lett. B 679, 19 (2009)

32. B. Singh, Nucl. Data Sheets 93, 33 (2001)

33. M. Danchev, et al., Phys. Rev. C 84, 061306 (2011)

34. G. Jakob, et al., Phys. Rev. C 65, 024316 (2002)

35. M. J. Bechara, O. Dietzsch, and J. H. Hirata, Phys. Rev. C 29, 1672 (1984)

36. S. Raman, C.W. Nestor Jr., and P. Tikkanen, At. Data \& Nucl. Data Tables 78, 1 (2001)
37. D. Bianco, F. Knapp, N. Lo Iudice, F. Andreozzi, and A. Porrino, Phys. Rev. C 85, 014313 (2012)

38. N. Lo Iudice, Ch. Stoyanov, and D. Tarpanov, Phys. Rev. C 77, 044310 (2008)

39. Ch. Stoyanov, QPM calculations for ${ }^{140} \mathrm{Nd}$, private communication (2012)

40. D. Bianco, F. Andreozzi, N. Lo Iudice, A. Porrino, and F. Knapp, Phys. Rev. C 84, 024310 (2011)

41. K. Gladnishki, et al., Phys. Rev. C 82, 037302 (2010) 